Original Paper http://ajol.info/index.php/ijbcs $\quad$ http://indexmedicus.afro.who.int

\title{
Effet de la phytoaccumulation du plomb, du fer et du zinc sur deux Poacées dans la zone de Kribi et de ses environs
}

\author{
Benjamin Obiang ONDO ${ }^{1}$, Sylvie Carole Ondo NTYAM ${ }^{2}$ et Richard Jules PRISO ${ }^{1 *}$ \\ ${ }^{1}$ Laboratoire de Biologie et de Physiologie des Organismes Végétaux, Faculté des Sciences, Université de \\ Douala. BP 24157 Douala, Cameroun. \\ ${ }^{2}$ Centre de Recherche sur les Ecosystèmes Marins / Institut de Recherche Agricole pour le Développement, \\ Kribi, Cameroun. \\ *Auteur correspondant ; E-mail : r_priso@yahoo.fr; Tél :00237677815765
}

\section{RESUME}

Le défi de préservation de la biodiversité reste pertinent en zones côtières notamment à Kribi, où la démographie galopante s'accompagne d'un accroissement notable des polluants. Pour appréhender les effets des métaux lourds dans le métabolisme de quelques macrophytes de Kribi, 4 sites de collecte des échantillons ont permis d'identifier deux Poacées, Panicum maximum et Echinochloa colona. Avec une température moyenne d'eau de $25,25 \pm 0,17^{\circ} \mathrm{C}, \mathrm{n}=16$; l'on constate la sensibilité thermique des feuilles de $E$. colona par rapport à $P$. maximum. Le site kribi plus acide $(\mathrm{pH}=4,16 \pm 0,02)$ contient aussi plus de plomb $(1,04 \pm 0,01 \mathrm{mg} / \mathrm{l})$. Corrélée au taux de chlorophylle et au nombre de feuilles de P. maximum, la quantité de chlorophylle accumulée et le nombre de feuilles diminuent avec l'augmentation du plomb. Ce métal provoque la chlorose foliaire, des lésions nécrotiques et le dessèchement du feuillage pour ces deux Poacées. Le fer s'observe plus dans le cours d'eau Bidou et augmente avec le taux de chlorophylle de $P$. maximum. La variation moyenne du zinc dans l'étude est de $0,11 \mathrm{mg} / \mathrm{l}$. L'accumulation du zinc empêche le développement de feuilles de P. maximum.

(C) 2020 International Formulae Group. All rights reserved.

Mots clés: Chlorophylle, Kribi, métaux lourds, Poacées, polluants.

\section{Effect of phytoaccumulation of lead, iron, zinc on Poaceae in Kribi and its surroundings}

\begin{abstract}
The challenge of preserving biodiversity in the context of modernization of industries remains relevant in coastal areas, especially in Kribi, where demography is galloping while pollutants increase. To control the effects of heavy metals in the metabolism of some macrophytes in Kribi, four sample collection sites were identified with two Poaceae, Panicum maximum and Echinochloa colona. With an average water temperature of $25.25 \pm 0.17{ }^{\circ} \mathrm{C}, \mathrm{n}=16$; the thermal sensitivity of $E$. colona leaves is found in relation to $P$. maximum. The more acidic Kribi site $(\mathrm{pH}=4.16 \pm 0.02)$ also contains more lead $(1.04 \pm 0.01 \mathrm{mg} / \mathrm{l})$. Correlated with chlorophyll and leaves of $P$. maximum, the amount of chlorophyll accumulated and the number of leaves decrease with increasing lead. This metal causes leaf chlorosis, necrotic lesions, yellowing and foliage drying, for these two Poaceae. Iron is observed more in Bidou stream and increases with maximum P. chlorophyll. The average variation of zinc in the study is $0.11 \mathrm{mg} / 1$ and the accumulation of zinc prevents the development of $P$. maximum leaves.
\end{abstract} (C) 2020 International Formulae Group. All rights reserved.

Keywords: Chlorophyll, heavy metals, Kribi, Poaceae, pollutants. 


\section{INTRODUCTION}

Les zones littorales sont davantage envahies par les polluants de tous ordres et notamment anthropiques et industriels (Douagui et al., 2009). La circulation des polluants dans les écosystèmes aquatiques favorise la phytoaccumulation qui permet d'évaluer la contamination (Soro et al., 2009).

Dans les villes, les ressources utilisées augmentent et conséquemment la production des déchets qui en découlent. L'industrialisation est croissante et de plus en plus productrice de polluants, sources de disfonctionnements divers des écosystèmes (Adjiri et al., 2008). La ville côtière de Kribi est victime ces dernières années des polluants industriels et anthropiques, pourtant cette cité balnéaire reste un espoir de pôle d'industrialisation en perpétuelle croissance.

La pollution des écosystèmes est à la base de plusieurs blocages du métabolisme des végétaux et à travers les chaînes trophique et alimentaire, la santé des populations environnantes est menacée (Aduayi-Akue et al., 2014). En effet, la plante accumule certains polluants à partir des racines, puis la tige et enfin les feuilles (Senou et al., 2012). Ces plantes sont consommées par les animaux et/ou les Humains (Kouakou et al., 2012) qui, par le phénomène de biomagnification (Hima et al., 2007), se retrouvent plus affectés par les métaux dans leur corps, engendrant de nombreuses maladies telles que les cancers, arrêts cardiaques, maladies odonto stomatologiques (Massaquoi et al., 2015). L'objectif global de l'étude est d'identifier l'influence de l'accumulation des éléments traces (plomb, fer et zinc) sur les macrophytes de la zone de Kribi et de ses environs.

\section{MATERIEL ET METHODES}

\section{Présentation de la zone d'étude}

Les sites de collecte des données se trouvent dans les arrondissements de Kribi (2 sites), de Lokoundjé (1 site) et de Nye'été (1 site), respectivement à $163 \mathrm{~km} ; 149 \mathrm{~km}$ et 182 $\mathrm{km}$ d'Ebolowa, la capitale régionale du Sud. Le Tableau 1 signale les coordonnées géographiques respectives des différents sites.
Le climat de la zone d'étude est équatorial, caractérisé par une grande saison de pluies dont les pics sont en septembre et octobre et une petite saison de pluie qui ne dépasse pas $300 \mathrm{~mm}$ de pluie au mois de mars. Une grande saison sèche va de décembre à février et une petite saison sèche est signalée au mois de juillet (moins de $130 \mathrm{~mm}$ ). Les précipitations qui atteignent des valeurs mensuelles de $504 \mathrm{~mm}$ en septembre à Kribi alors que des pics de température mensuelle de $28,2{ }^{\circ} \mathrm{C}$ sont notés en février et mars. Les sols sont ferralitiques jaunes, perméables, très pauvres en matière organique, un $\mathrm{pH}$ acide, avec faible capacité d'échanges à cause de sa richesse en hydroxyde de fer et d'aluminium.

$\mathrm{La}$ végétation riche et variée est constituée de forêts à marais, de forêts à mangroves et de forêts à feuilles persistantes. L'ensemble de cette végétation crée un milieu spécifique à la plage, ce qui la rend attractive pour les touristes.

Le réseau hydrographique de Kribi est dominé par l'océan atlantique dont l'amplitude des marées est basse avec de petites vagues. La présence des fleuves lobé, Kiénké et Nyong est remarquable de même que les rivières Ngoye, Abondé, Wamié, Nzami, Bidou, Nyé'été, lesquelles reçoivent les déchets ménagers et ceux des sociétés agroindustrielles (Socapalm et Hevecam). Les températures de surface sont généralement au-dessus de $25^{\circ} \mathrm{C}$.

\section{Matériel, méthode et choix du site d'étude}

Les bouteilles plastiques de $33 \mathrm{cl}$ ont été utilisées pour prélever l'eau préalablement troublée et placées dans une glacière contenant de la glace. Une pirogue et des perches ont été utilisées pour des prélèvements éloignés de la rive, les voies d'accès étant ouvertes à l'aide de la machette.

- Le laboratoire de l'Institut de Recherche Agricole pour le Développement (IRAD) à Yaoundé a été utile pour obtenir les paramètres physico-chimiques des eaux et le laboratoire du Département de Biologie des Organismes Végétaux de l'Université de Douala a servi pour le dosage de la chlorophylle. 
- Le pHlthermomètre de marque Hanna HI98127 a servi pour les valeurs de $\mathrm{pH}$ et de température.

- Le conductimètre de marque Hanna modèle HI8724 a permis d'avoir les valeurs de la conductivité (CND) et celles des Matières Totales Dissoutes (MTD ou STD).

Le choix des sites s'est opéré après une prospection sur le terrain, laquelle a permis d'apprécier la qualité du milieu :

- une première rivière a été retenue comme site témoin, parce que loin des habitations et de tout déversement d'effluents susceptible d'altérer la qualité de ses eaux (Kienké à Mimbougué) et correspondant à $\mathrm{P} 1$;

- dans une seconde rivière, l'on observe que les effluents d'huile de palme traités par l'agroindustrie SOCAPALM y sont déversés (rivière Bidou) et correspond à $\mathrm{P} 2$;

- une troisième rivière retenue à proximité des plantations d'hévea, dans laquelle est déversée une importante quantité de pesticides utilisés dans lesdites plantations (rivière Nye'été) et correspond à $\mathrm{P} 3$;

- une quatrième rivière où l'on note des déversements dans son lit des déchets d'une scierie de bois et des déchets domestiques (rivière Abondé à Kribi) a été retenue et correspond à $\mathrm{P} 4$.

\section{Analyse des composantes biologiques et inventaire des espèces végétales}

Des relevés floristiques ont été élaborés afin d'inventorier le nombre d'espèces et leur densité. Une ficelle a été utilisée pour délimiter les parcelles. Un couteau tranchant a été utilisé pour découper les organes de la plante. Un mètre ruban et une règle graduée de $10 \mathrm{~cm}$ ont permis les mensurations de la taille de la tige, taille de la racine, nombre de fruits, largeur et longueur des feuilles et mesure des parcelles. Ainsi, sur chaque site une parcelle de $20 \mathrm{~m}^{2}$ soit (4 m x $5 \mathrm{~m}$ ) est délimitée sur une berge, les espèces recensées sont accompagnées d'un coefficient d'Abondance-Dominance (A-D) pour estimer le nombre d'individus et surface de recouvrement, pourcentage de recouvrement spatial de chacune d'elle suivant l'échelle de Braun-Blanquet (Tableau 2).
Les formules suivantes ont été utilisées :

Le recouvrement moyen $\left(\mathrm{RM}_{\mathrm{i}}=\mathrm{R}_{\mathrm{i}} / \mathrm{N}_{\mathrm{t}}\right)$ Avec $\mathrm{RM}_{\mathrm{i}}=$ recouvrement moyen de l'espèce i.

Et l'indice de diversité de Shannon Weaver $\mathrm{H}$ $=-\Sigma \mathrm{P}_{\mathrm{i}} \times \ln \left(\mathrm{P}_{\mathrm{i}}\right)$ avec $\mathrm{P}_{\mathrm{i}}=\mathrm{N}_{\mathrm{i}} / \mathrm{N}$, où $\mathrm{P}_{\mathrm{i}}$ est la probabilité d'occurrence des individus de l'espèce i. L'indice de régularité $\mathrm{R}^{\prime}=\mathrm{H} /$ H'max, où $H^{\prime} \max =$ la diversité maximale ou l'équifréquence $=\ln S(\mathrm{~S}=$ Nombre d'espèces $=$ Richesse spécifique).

\section{Echantillonnage du matériel végétal}

Les paramètres suivants ont été pris: la taille de la plante, la longueur des feuilles, la largeur des feuilles et le nombre des racines par plante. Pour calculer la surface foliaire, l'on a utilisé la formule : Surface $(S)=2 / 3 \times$ longueur (Lf) $x$ largeur (lf).

Le Choix du matériel végétal de cette étude a porté sur plusieurs critères qui englobent la variété des espèces végétales, l'aspect morphologique, ainsi que physiologique et leur comportement vis-à-vis des changements du milieu environnant. Les espèces échantillonnées sont essentiellement, Echinochloa colona et Panicum maximum.

Le dosage de la chlorophylle a fait appel à un spectrophotomètre HACH DR/3900. Une balance numérique de haute précision tactile ( 5 $\mathrm{kg} / \mathrm{g}$ ) de marque REIDEA a été utilisée pour obtenir le poids des feuilles. L'acétone à $80 \%$ a pour favoriser l'extraction du liquide. Le sable fin $(30 \mathrm{cl})$ a été nécessaire pour l'émiettage des feuilles dans un mortier en porcelaine dans lequel les feuilles ont été broyées avec un petit pilon.

Pour analyser la teneur en chlorophylle des plantes, un échantillonnage a été réalisé sur les feuilles. Des feuilles de même âge (4 à 5 semaines) des plantes ont été lavées et essuyées. Puis coupées à l'aide d'un couteau pour ensuite être pesées, à l'aide d'une balance de précision pour obtenir $0,80 \mathrm{~g}$ de matière fraîche. Ce matériel végétal a été broyé dans un mortier en porcelaine avec du sable lavé, séché et stérilisé. Dix millilitres d'acétone à $80 \%$ sont ajoutés dans le mélange et l'extrait est ensuite centrifugé pendant $10 \mathrm{mn}$. Le surnageant est alors récupéré et le culot est repris dans $10 \mathrm{ml}$ 
d'acétone à $80 \%$ puis centrifugé à nouveau afin d'extraire toute la chlorophylle.

La chlorophylle a été extraite et analysée dans le Laboratoire du Département de Biologie des Organismes Végétaux de l'Université de Douala.

Pour le prélèvement des eaux, au niveau de chaque rivière, il a été délimité un carré de $16 \mathrm{~m}^{2}$. Un total de 8 prélèvements aléatoires a été effectué selon les quadrats, près de la rive, au milieu de la rivière et toujours au point de la pollution.

Des bouteilles plastiques de $33 \mathrm{cl}$ ont servi pour prélever l'eau préalablement troublée. Le $\mathrm{pH} \backslash$ thermomètre de marque Hanna HI98127 a servi pour les valeurs de $\mathrm{pH}$ et de température. Le conductimètre de marque Hanna modèle HI8724 a permis d'avoir les valeurs de la conductivité (CND) et celles des matières Totales Dissoutes (MTD ou STD).

Le dosage de l'eau a permis d'identifier les différents polluants de l'écosystème étudié. Les paramètres physico-chimiques des eaux ont été analysés au laboratoire de l'Institut de Recherche Agricole pour le Développement
(IRAD) à Yaoundé. Après acidification des échantillons d'eau et vérification que le $\mathrm{pH}$ est inférieur à 2 , le dosage des éléments traces dans les échantillons d'eau a été fait par la méthode d'absorption atomique ICP (NF EN ISO 11885, 1998).

Ainsi, un thermomètre approprié (à électrodes) a été utilisé en plongeant ses électrodes dans de l'eau du cours d'eau, en conformité avec Rodier et al. (2005); la valeur de la température est exprimée en degrés Celsius; le $\mathrm{pH}$ a été mesuré avec un $\mathrm{pH}$-mètre, composé d'une électrode de verre, d'une électrode de référence au calomel-KCl saturé et d'un dispositif potentiométrique.

Les éléments traces métalliques (Cadmium, Plomb, Fer, Zinc) ont été déterminés par la méthode d'absorption atomique. Le Cadmium complexe par l'ammonium pyrrolidium dithiocarbamate (APDC) est extrait à un pH égal à 3,5 par la methyl-isobutylcetone puis dosé par spectrométries d'absorption atomique directement sur la solution obtenue (Rodier, 1984).

Tableau 1: Coordonnées géographiques des sites de collecte des données.

\begin{tabular}{lll}
\hline Sites et points (P) de collecte des eaux & Coordonnées géographiques (UTM) \\
\hline Kienké (Mimbougué) P1 & N $0604470 ;$ & E 0326944 \\
\hline Nye'été (Hévécam) P2 & N $0614525 ;$ & E 0307937 \\
\hline Bidou (Socapalm) P3 & N 0608342; & E 0315079 \\
\hline Abondé (Kribi centre) P4 & N $0602337 ;$ & E 0326086 \\
\hline
\end{tabular}

Tableau 2: Abondance-Dominance et recouvrements moyens des macrophytes.

\begin{tabular}{cccc}
\hline $\begin{array}{c}\text { Abondance-Dominance } \\
\text { (A-D) }\end{array}$ & $\begin{array}{c}\text { Classe de Recouvrement } \\
\text { (en \%) }\end{array}$ & $\begin{array}{c}\text { Recouvrement } \\
\text { moyen (RM) }\end{array}$ & $\begin{array}{c}\text { Type de } \\
\text { recouvrement }\end{array}$ \\
\hline $\mathbf{5}$ & $75-100$ & 87,5 & Très élevé \\
\hline $\mathbf{4}$ & $50-75$ & 62,5 & Elevé \\
\hline $\mathbf{3}$ & $25-50$ & 37,5 & Moyen \\
\hline $\mathbf{2}$ & $5-25$ & 15 & Faible \\
\hline $\mathbf{1}$ & $1-5$ & 3 & Assez faible \\
\hline+ & $<1$ & 0,5 & Très faible \\
\hline
\end{tabular}



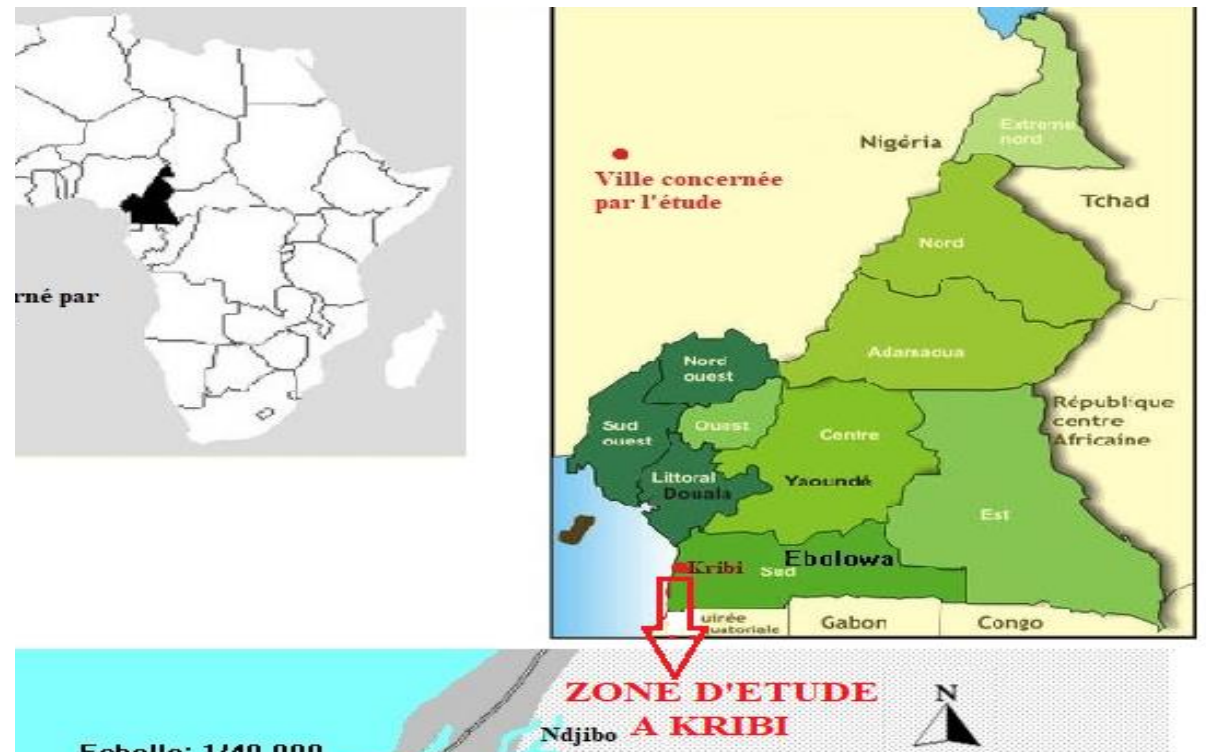

Echelle: 1/40 000 Ndjibo A KRIBI

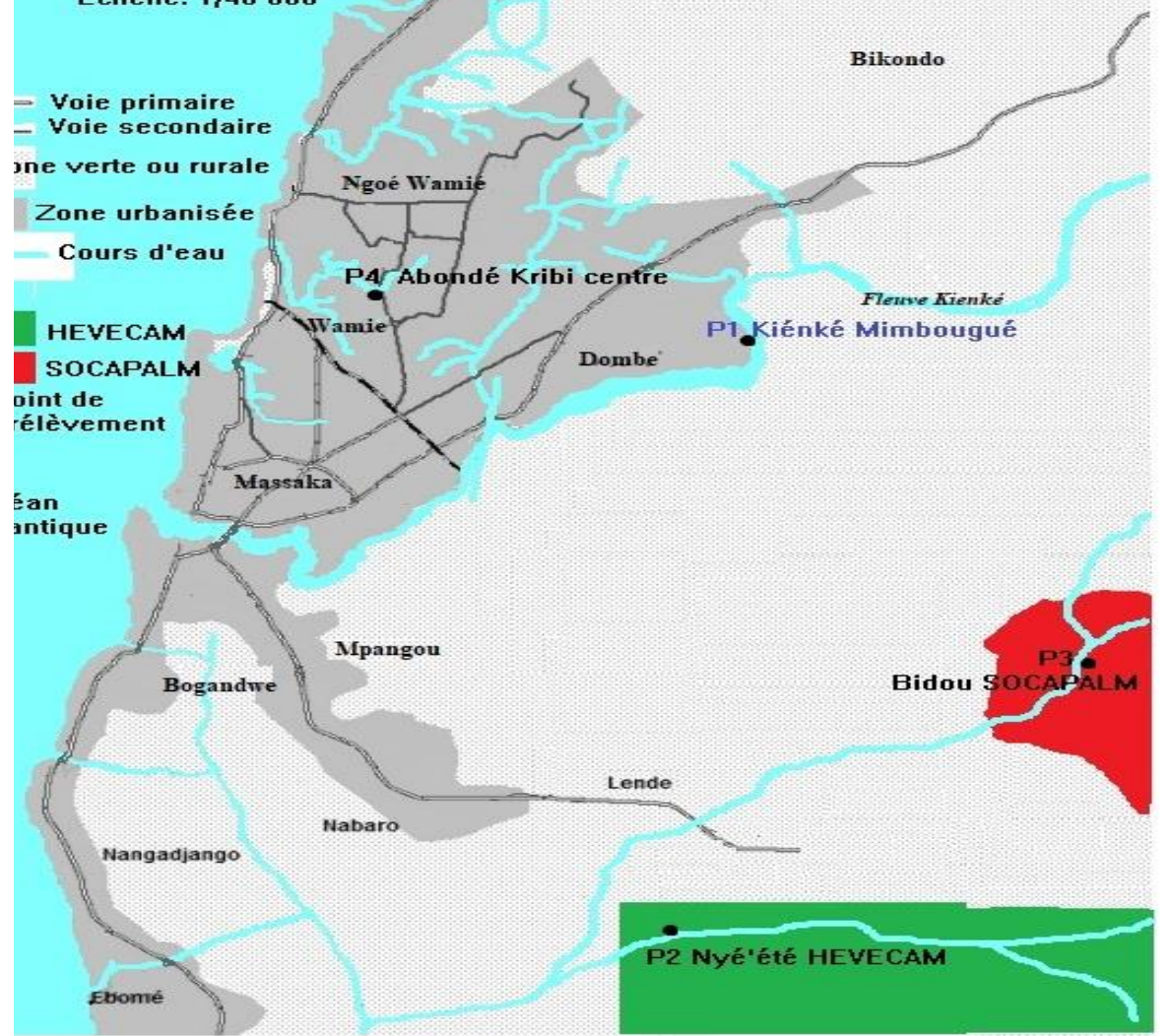

Figure 1: Localisation du site. 
Tableau 3: Valeurs des paramètres physico-chimiques étudiés.

\begin{tabular}{|c|c|c|c|c|c|c|c|}
\hline Echantillon $n=16$ & $\mathbf{p H}$ & $\mathbf{T}\left({ }^{\circ} \mathbf{C}\right)$ & Salinité & $\mathrm{Fe}(\mathrm{mg} / \mathrm{l})$ & $\mathrm{Zn}(\mathrm{mg} / \mathrm{l})$ & $\begin{array}{c}\text { Cd } \\
(\mathrm{mg} / \mathrm{l})\end{array}$ & $\mathrm{Pb}(\mathrm{mg} / \mathrm{l})$ \\
\hline Kiénké (P1) & $7,18 \pm 0,00$ & $\begin{array}{c}25,40 \pm \\
0,24\end{array}$ & 0 & $\begin{array}{c}1,70 \pm \\
0,11\end{array}$ & $\begin{array}{c}0,12 \pm \\
0,00\end{array}$ & 0 & $\begin{array}{c}0,29 \pm \\
0,01\end{array}$ \\
\hline Nye'été (P2) & $6,78 \pm 0,01$ & $\begin{array}{c}25,21 \pm \\
0,21\end{array}$ & 0 & $\begin{array}{c}0,00 \pm \\
0,00\end{array}$ & $\begin{array}{c}0,03 \pm \\
0,00\end{array}$ & 0 & $\begin{array}{c}0,04 \pm \\
0,00\end{array}$ \\
\hline Bidou (P3) & $5,09 \pm 0,02$ & $\begin{array}{c}25,10 \pm \\
0,08\end{array}$ & 0 & $\begin{array}{c}2,64 \pm \\
0,17\end{array}$ & $\begin{array}{c}0,18 \pm \\
0,00\end{array}$ & 0 & $\begin{array}{c}0,46 \pm \\
0,00\end{array}$ \\
\hline Abondé (P4) & $4,16 \pm 0,02$ & $\begin{array}{c}25,28 \pm \\
0,15\end{array}$ & 0 & $\begin{array}{c}0,61 \pm \\
0,01\end{array}$ & $\begin{array}{c}0,14 \pm \\
0,01\end{array}$ & 0 & $\begin{array}{c}1,04 \pm \\
0,01\end{array}$ \\
\hline $\begin{array}{c}\text { Moyenne globale des } \\
\text { sites }\end{array}$ & $5,34 \pm 1,33$ & $\begin{array}{c}25,20 \pm \\
0,09\end{array}$ & 0 & $\begin{array}{c}1,08 \pm \\
1,38\end{array}$ & $\begin{array}{c}0,11 \pm \\
0,07\end{array}$ & 0 & $\begin{array}{c}0,51 \pm \\
0,50\end{array}$ \\
\hline Norme Camerounaise & $6-9$ & $30-35$ & & 1 & $1-3$ & $<0,1$ & 1 \\
\hline
\end{tabular}

Tableau 4: Valeurs des corrélations entre paramètres physico chimiques.

\begin{tabular}{lccccc}
\hline Corrélations $(\mathbf{r})$ & $\mathbf{p H}$ & $\mathbf{T}\left({ }^{\circ} \mathbf{C}\right)$ & $\mathbf{F e}(\mathbf{m g} / \mathbf{l})$ & $\mathbf{Z n}(\mathbf{m g} / \mathbf{l})$ & $\mathbf{P b}(\mathbf{m g} / \mathbf{l})$ \\
\hline $\mathrm{pH}$ & 1,00 & & & & \\
\hline $\mathrm{T}^{\circ} \mathrm{C}$ & 0,42 & 1,00 & & & \\
\hline $\mathrm{Fe}(\mathrm{mg} / \mathrm{l})$ & $-0,11$ & $-0,25$ & 1,00 & & \\
\hline $\mathrm{Zn}(\mathrm{mg} / \mathrm{l})$ & $-0,56$ & $-0,16$ & $0,84^{* *}$ & 1,00 & \\
\hline $\mathrm{Pb}(\mathrm{mg} / \mathrm{l})$ & $-0,88^{*}$ & 0,05 & 0,06 & 0,59 & 1,00 \\
\hline${ }^{*}$ Corrélative négative significative & ** Corrélation positive significative & &
\end{tabular}

Tableau 5: Valeurs des corrélations entre paramètres physico chimiques.

\begin{tabular}{|c|c|c|c|c|c|c|c|c|c|c|}
\hline & \multicolumn{2}{|c|}{ Chlorophylle } & \multicolumn{2}{|c|}{ Surface foliaire } & \multicolumn{2}{|c|}{ Longueur tige } & \multicolumn{2}{|c|}{ Nombre feuilles } & \multicolumn{2}{|c|}{ Nombre racines } \\
\hline & $P$. & $E$. & $P$. & $E$. & $P$. & $E$. & $P$. & $E$. & $P$. & $E$. \\
\hline & maximum & colona & maximum & colona & maximum & colona & maximum & colona & maximum & colona \\
\hline $\mathrm{pH}$ & 0,38 & $0,99 * *$ & 0,35 & $-0,48$ & $-0,81 *$ & $0,81 * *$ & 0,66 & $-0,26$ & $-0,93 *$ & $0,81^{*}$ \\
\hline $\mathrm{T}^{\circ} \mathrm{C}$ & $-0,44$ & 0,32 & $0,99 * *$ & $-0,99 *$ & 0,05 & 0,15 & $-0,36$ & $0,76^{* *}$ & $-0,35$ & 0,27 \\
\hline $\mathrm{Fe}(\mathrm{mg} / \mathrm{l})$ & $0,73 * *$ & $-0,06$ & $-0,11$ & 0,14 & $-0,39$ & 0,48 & $-0,16$ & $-0,29$ & $-0,27$ & 0,50 \\
\hline $\mathrm{Zn}(\mathrm{mg} / \mathrm{l})$ & 0,27 & $-0,54$ & $-0,01$ & 0,11 & 0,16 & $-0,02$ & $-0,64$ & 0,13 & 0,21 & 0,03 \\
\hline $\mathrm{Pb}(\mathrm{mg} / \mathrm{l})$ & $-0,60$ & $-0,92 *$ & 0,13 & 0,01 & $0,89 * *$ & $-0,77 *$ & $-0,93^{*}$ & 0,67 & $0,81 * *$ & $-0,70$ \\
\hline
\end{tabular}


B. O. ONDO et al. / Int. J. Biol. Chem. Sci. 14(6): 2257-2266, 2020
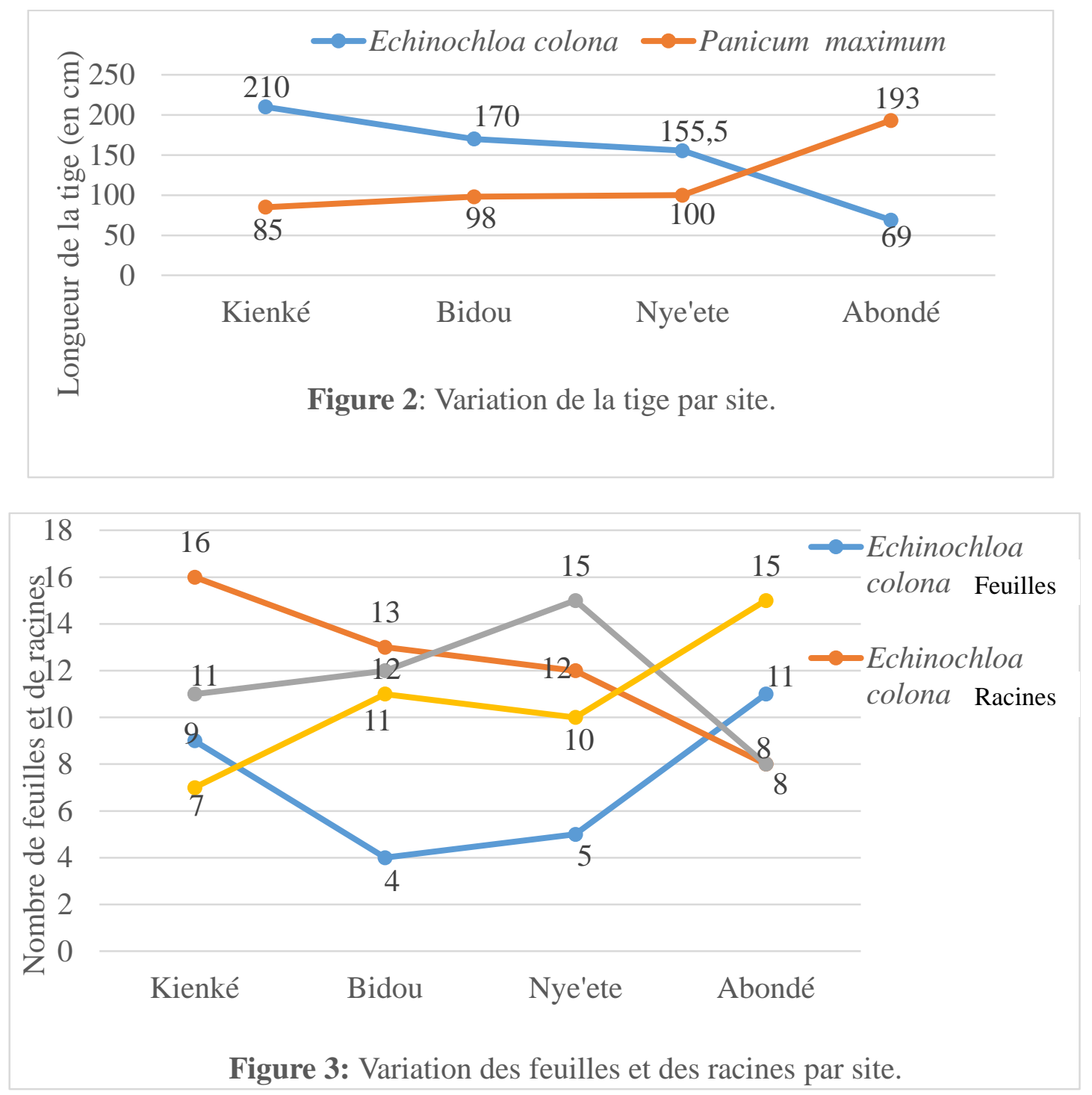

\section{RESULTATS}

Présentation des paramètres physicochimiques étudiés

\section{Inventaire des macrophytes}

Les inventaires des macrophytes ont permis de recenser 29 familles, 42 genres et 48 espèces. Les familles les plus représentatives sont : Acanthaceae; Amaranthaceae; Araceae; Arecaceae; Asteraceae; Capparidaceae; Caricaceae; Commelinaceae; Convovulaceae; Costaceae; Cyperaceae; Dennstaedtiaceae; Euphorbiaceae; Fabaceae; Lamiaceae; Lentibulariaceae; $\quad$ Malvaceae; Melaslomataceae; Mimosaceae; Musaceae; Nymphaeaceae; Ocynaceae; Onagraceae;
Oxalidaceae; Poaceae; Polygonaceae; Portulacaceae; Rhizophoraceae; Rubiaceae. Les espèces et genres les plus représentatifs sur les différents sites sont: Asystasia gangetica (L)T. Anders; Dicliptera verticillata (Forsk.) C. Christ; Amaranthus hybridus Linn; A. spinosus Linn; A.viridus Linn; Alstonia boonei De Wild; Manihot esculenta Crantz; Xanthosoma mafaffa L; Xanthosoma sagittifolium L.; Elaeis guineensis Jacq.; Acmella caulirhiza Del.; Ageratum conyzoides Linn; Galinsoga ciliata (Raf.) Blake; Melanthera elliptica O. Hoffm; Synedrella nodiflora Gaertn; Vernonia spp; Cleome ciliata L.; Carica papaya Linn; 
Commelina benghalensis Linn. Fam; Ipomoea batatas (L.) Lam; Ipomoea involucrala P. Beauv; Costus afer Ker GawI; Cyperus difformis L.; Pteridium aquilinum; Desmodium ramosissimum G. Don.; Pueraria javanica (Roxb.) Benth.; Solenostemon monoslachyus; Utricularia macrorhiza Leconte; Corchorus trilocularis L.; Dissotis rotundifolia (Sm.) Triana; Albizia laurentii De Wild; Musa paradisiaca; Nymphaea lotus Linn; Ludwigia palustris L.; Oxalis barrelieri L.; Acroceras amplectens Stapf.; A. zizanoides (Kunth.) Dandy; Bambusa Vulgaris; Echinochloa colona (Linn.) Link; E. pyramidalis (Lam.) Hitche \& Chardie; Eleusine indica L.; Paspalum dilatatum; Panicum maximum Jacq.; Setaria barbata Lam. Kunth; Polygonum acuminatum H.B.K.; Portulaca oleraceae L.; Rhizophora Svp.; Oldenlandia corymbosa.

Le plus grand nombre d'espèces ( 30 sur les 42 possibles) a été observé dans le site témoin qu'est Mimbougué (rivière Kienké) et dans la zone de Nye'été (29 espèces). Les sites les plus pollués présentent le moins de macrophytes (22 et 24 espèces) recensées respectivement à Socapalm et Kribi centre. L'indice de régularité est respectivement de 0,844 à Kribi centre; 0,885 à Socapalm et de 0,883 à Nye'été contre 0,70 dans le site de Mimbougué.

\section{Longueur de la tige et nombre de feuilles}

Les valeurs moyennes de la longueur des tiges de Echinochloa colona et de Panicum maximum sont respectivement de 131,33 \pm $54,50 \mathrm{~cm}$ et $130,33 \pm 54,28 \mathrm{~cm}$. Les tiges de Echinochloa colona sont les plus longues sur tous les sites (un maximum de $210 \mathrm{~cm}$ ) excepté celui le plus pollué Kribi centre (Abondé), où les tiges de P. maximum sont plus longues (193 $\mathrm{cm})$. Les tiges de Echinochloa colona mesurent $69 \mathrm{~cm}$ sur ledit site le plus pollué. La longueur de la tige de ce macrophyte baisse avec l'augmentation de la pollution en $\mathrm{Zn}, \mathrm{Fe}, \mathrm{Pb}$ contrairement aux tiges de $P$. maximum qui augmentent avec la pollution au $\mathrm{Pb}$. Le nombre moyen de feuilles observées d'Echinochloa colona est de 6,67 $\pm 3,79$ contre $11,67 \pm 3,51$ pour le Panicum maximum.

\section{DISCUSSION}

\section{Abondance et recouvrement}

L'analyse de la répartition des plantes montre une abondance de Poaceae, dans un gradient contraire à la pente de la berge du cours d'eau, avec notamment Panicum maximum, Echinochloa colona et Eleusine indica dans tous les 3 sites échantillonnés. Les nénuphars (Nymphaea lotus) sont observés sur deux sites, Kribi centre et Nye'été, avec les mêmes valeurs du recouvrement moyen $(\mathrm{Pi}=$ 0,094; $\mathrm{RM}=26,5$ ); ces macrophytes flottent sur l'eau et ceux du site de Kribi centre présentent des surfaces foliaires plus développées que les autres de la zones. Ces résultats sont en accord avec ceux de Barendregt et Bio (2003).

\section{Le pH, la température et les métaux lourds}

Le $\mathrm{pH}$ moyen des sites $(5,3 \pm 1,33)$ indique une acidité, caractéristique des eaux très polluées. Corrélé au plomb $(\mathrm{r}=-0,86)$, pour $74 \%$ d'observations, le $\mathrm{pH}$ favorise l'accumulation $\mathrm{du} \mathrm{Pb}$ qui inhibe la production de la chlorophylle (98\% de cas). La chlorophylle b est la plus accumulée sur les sites par E. colona $(31,73 \pm 1,33 \mathrm{mg} / \mathrm{l})$ contre $17,44 \pm 0,98 \mathrm{mg} / \mathrm{l}$ pour $P$. maximum. Ces résultats sont en accord avec Priso et al. (2010) puis Senou et al. (2012).

La quantité de fer est la plus élevée à Socapalm $(2,64 \pm 0,17 \mathrm{mg} / \mathrm{l})$, une pollution très importante. Il s'agit des rejets de débris de fer dans les effluents déversés dans la nature par les huileries. Le fer $(r=0,73)$ stimule l'accumulation ou la production de la chlorophylle dans 53\% de cas. En effet, il favorise l'accumulation $\mathrm{du}$ phosphate $(\mathrm{r}=$ $0,80)$, qui stimule à son tour la production de la chlorophylle de P. maximum ( $64 \%$ cas). Ce résultat corrobore ceux de Kabata-Pendias et Pendias (2001), Chouiti et al. (2018), Vaňková et al. (2018).

Le $\mathrm{Pb}$ est négativement corrélé à la chlorophylle $(r=-0,60)$, et aux feuilles $(r=-$ $0,93)$ de $P$. maximum. L'on constate une inhibition de la chlorophylle et des feuilles ( $86 \%$ de cas). C'est le même effet sur $E$. colona, pour la chlorophylle $(\mathrm{r}=-0,92)$, soit $84 \%$ de cas; la longueur de la tige $(r=-0,77)$, puis le nombre de racines $(r=-0,70)$. Ces corrélations et coefficients de détermination 
indiquent que le plomb est la cause de la chlorose foliaire, des lésions nécrotiques, jaunissement progressif, du repliement ou du dessèchement du feuillage pour ces deux Poacées. Il bloque ainsi leur métabolisme, ce qui confirme les travaux de Mununga et al. (2018). A forte concentration, il agit comme un facteur de stress et il altère le développement et la croissance normale des plantes (Chouti et al., 2018). Le Plomb est d'ailleurs sans effet bénéfique même à de très faibles concentrations dans un macrophyte (KabataPendias et Pendias, 2001; Garnier, 2005).

L'accumulation du zinc inhibe le développement du nombre de feuilles de $P$. maximum $(\mathrm{r}=-0,64)$ et la production de la chlorophylle de E. colona $(\mathrm{r}=-0,54)$. Toutefois, le fait que seulement $30 \%$ de cas détermine ce résultat dans l'étude, suppose que d'autres facteurs interfèrent avec du zinc pour réduire aussi bien la production de la chlorophylle que le nombre de feuilles (Kamal et al., 2004).

\section{Conclusion}

Le $\mathrm{pH}$ moyen du site est de 5,3 $\pm 1,33$. Cette acidité importante influe sur la phytoaccumulation du zinc et du plomb qui contribue au blocage de la production de la chlorophylle et l'inhibition de la production des feuilles de $P$. maximum et $E$. colona. Sur $E$. colona, ces métaux inhibent la croissance de la tige et l'enracinement avec pour conséquence la chlorose et le rabougrissement des plantes. La chaleur est un facteur de stress pour les feuilles de E. colona.

\section{CONFLIT D'INTERETS}

Les auteurs déclarent ne pas avoir de conflits d'intérêts.

\section{CONTRIBUTIONS DES AUTEURS}

BOO a collecté, analysé, interprété les données primaires et a rédigé le manuscrit. SCON a participé au recadrage de la problématique, à la consolidation de la méthodologie et à relecture des résultats. RJP a participé à la reformulation de la méthodologie, des résultats, à l'interprétation des données statistiques et à la relecture du manuscrit.

\section{REMERCIEMENTS}

Les remerciements des auteurs vont principalement aux responsables et encadreurs du Laboratoire de Biologie et de Physiologie des Organismes Végétaux de l'Université de Douala au Cameroun.

\section{REFERENCES}

Adjiri OA, Gone DL, Kouame IK, Kamagate B, Biemi J. 2008. Caractérisation de la pollution chimique et microbiologique de l'environnement de la décharge d'Akouédo, Abidjan-Côte d'Ivoire. Int. J. Biol. Chem. Sci., $\mathbf{4}(2)$. DOI: https://dx.doi.org/10.4314/ijbcs.v2i 4.39768.

Aduayi-Akue AA, Gnandi K. 2014. Evaluation de la pollution par les métaux lourds des sols et de la variété locale du maïs Zea mays dans la zone de traitement des phosphates de Kpémé (Sud du Togo). Int. J. Biol. Chem. Sci., 8(5): 2347-2355. DOI: https://dx.doi.org/10.4314/ijbcs.v8i 5.37 .

Barendregt A, Bio AMF. 2003. Relevant variables to predict macrophyte communities in running waters. Ecological Modelling, 160(3): 205-217. DOI: https://dx.doi.org/10.1016/S03043800 (02)00254-5.

Chouti WK, Adanvei, Mama D. 2018. Dosage du plomb et du zinc dans les cultures de l'amarante (Amarantus cruentus) et de la Grande morelle (Solanum macrocarpum): cas de quelques sites maraîchers de Porto-Novo. Int. J. Biol. Chem. Sci., 12(5): 2381-2395. DOI: https://dx.doi.org/10.4314/ijbcs.v12i5.36

Douagui AG, Kouame IK, Dibi B, Konan FK, Savane I. 2009. Origines et modélisation de la minéralisation des eaux du Quaternaire d'Abidjan (Sud de la Côte d'Ivoire). Int. J. Biol. Chem. Sci., 3(5): 856-869.

DOI: https://dx.doi.org/10.4314/ijbcs.v3i5.510 49.

Garnier R. 2005. Toxicité du plomb et de ses dérivés. EMC (Elsevier SAS, Paris). Toxicologie-Pathologie Professionnelle, 10: 1-22. DOI: https://dx.doi.org/10.1016/S11551925(05)38475-8. 
Hima KA, Srinivasa RR, Vijaya SS, Jayakumar SB, Suryanarayana V, Venkateshwar P. 2007. Biosorption: An eco-friendly alternative for heavy metal removal. African Journal of Biotechnology, 6(25): 2924-2931. https://dx.doi.org/10.5897/AJB2007.000 $-2461$.

Kabata-Pendias A, Pendias H. 2001. Trace Elements in Soils and Plants ( ${ }^{\text {rd }}$ edn) CRC Press: Boca Raton, London, NewYork, Washington D.C.

Kamal M, Ghaly AE, Mahmoud N, Côtė R. 2004. Phytoaccumulation of heavy metals by aquatic plants. International Environment, 29: 1029-1039. DOI: https://dx.doi.org/10.1016/S01604120(03)00091-6.

Kouakou KJ, Sika AE, Denezon D, Bekro YA, Baize D, Bounakhla M, Zaher F et Tahri M. 2012. Teneurs de métaux traces dans des sols à maraîchers dans la ville d'Abidjan (Côte d'Ivoire). Int. J. Biol. Chem. Sci., 6(5): 2252-2262. DOI: https://dx.doi.org/10.4314/ijbcs.v6i 5.31 .

Massaquoi LD, Ma H, Liu XH, Han PY, Zuo SM, Hua ZX, Liu DW. 2015. Heavy metal accumulation in soils, plants, and hair samples: an assessment of heavy metal exposure risks from the consumption of vegetables grown on soils previously irrigated with wastewater. Environ. Sci. Pollut. Res., 22 : $18456-$ 18468.

DOI: https://dx.doi.org/10.1007/S11356-0155131-1.

Mununga KF, Mwilambwe KX, N'tambwe ND, Maloba KJP, Banza IM, Chola K A, Lwanga KM, Mpundu MM. 2018. Vigueur de croissance de deux variétés de légumes installées sur sols contaminés à Lubumbashi (RD Congo). J. Appl. Biosci., 124: 12396-12407. DOI: https://dx.doi.org/10.4314/jab.v124i1.1.

NF EN ISO 11885. 1998. Qualité de l'eauDosage de 33 éléments par spectrométrie d'émission atomique avec plasma couplé par induction.
Priso RJ, Dibong SD, Tchinda MC, Taffouo VD, Din N, Amougou A. 2010. Impacts des eaux polluées sur la croissance, les teneurs en chlorophylles et substances organiques dans les feuilles de deux Poaceae. Int. J. Biol. Chem. Sci., 4(4): 273-280.

DOI: https://dx.doi.org/10.4314/ijbcs.v4i4.630 49.

Rodier J. 1984. L'Analyse de l'Eau : Eaux Naturelles, Eaux Résiduaires Eaux de Mer (7e édn). Dunod : Paris ; 1365 p.

Rodier J, Bazinc AC, Broutin JP, Chambon P, Champsaur H, Rodier L. 2005. L'Analyse de l'Eau: Eaux Naturelles et Eaux Résiduaires et Eau de Mer (8 édn). Dunod : $1384 \mathrm{p}$.

Senou I, Gnankambary Z, Some AN, Sedogo MP. 2012. Phytoextraction du cadmium, du cuivre, du plomb et du zinc par Vetiveria nigritana en sols ferrugineux tropicaux et en sols vertiques au Burkina Faso (Afrique de l'ouest). Int. J. Biol. Chem. Sci., 6(4): 1437-1452. DOI: https://dx.doi.org/10.4314/ijbcs.v6i4.4.

Soro G, Metongo BS, Ahoussi EK, Kouamé FK, Zade PG, Soro T. 2009. Métaux lourds $(\mathrm{Cu}, \mathrm{Cr}, \mathrm{Mn}$ et $\mathrm{Zn})$ dans les sédiments de surface d'une lagune tropicale africaine : cas de la lagune Ebrie (Côte d'Ivoire). Int. J. Biol. Chem. Sci., 3(6): 1408-1427. DOI: https://dx.doi.org/10.4314/ijbcs.v3i6.531 61.

Tchiaze IAV, Priso RJ. 2016. Répartition et valorisation des macrophytes envahissantes dans la region du littoral (Cameroun) : cas d'Eichhornia crassipes (Mart.) Solms-Laubach. J. Appl. Biosci., 100: 9522-9534. DOI: https://dx.doi.org/10.4314/jab.v100i1.4.

Vaňková K, Marková I, Jašprová J, Dvořák A, Subhanová I, Zelenka J, Vítek L. 2018. Chlorophyll-Mediated Changes in the Redox Status of Pancreatic Cancer Cells Are Associated with Its Anticancer Effects. Oxidative Medicine and Cellular Longevity.

DOI: https://dx.doi.org/10.1155/2018/4069167 